\title{
TBL1XR1 as a potential therapeutic target that promotes epithelial-mesenchymal transition in lung squamous cell carcinoma
}

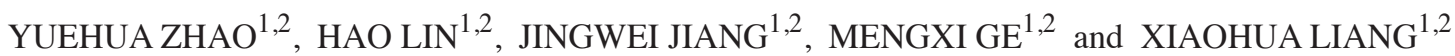 \\ ${ }^{1}$ Department of Oncology, Huashan Hospital, Fudan University; ${ }^{2}$ Department of Oncology, \\ Shanghai Medical College of Fudan University, Shanghai 200040, P.R. China
}

Received May 25, 2018; Accepted August 22, 2018

DOI: $10.3892 /$ etm.2018.6955

\begin{abstract}
Transducin ( $\beta$ )-like 1 X-linked receptor 1 (TBL1XR1) has been demonstrated to serve a vital role in tumor progression. However, the biological role and molecular mechanisms of TBL1XR1 in lung squamous cell carcinoma (SCC) remain largely unknown. The purpose of the present study was to investigate the biological role of TBL1XR1 and its mechanism in lung SCC. TBL1XR1 was expressed in a human bronchial epithelial cell line and in lung SCC cell lines. The present study analyzed TBL1XR1-induced proliferation, invasion and migration abilities in vitro using the cell counting kit- 8 assay, cell invasion assay and wound healing assay, respectively. This study examined the effects of TBL1XR1 on epithelial-mesenchymal transition (EMT) in lung SCC cells and activation of the transforming growth factor (TGF)- $\beta /$ mothers against decapentaplegic homolog (Smad) signaling pathway by western blotting. The results indicated that TBL1XR1 was upregulated in lung SCC cells. Overexpression of TBL1XR1 increased the rate of cell proliferation compared with the control group. In vitro, overexpression of TBL1XR1 promoted cell invasion and migration ability compared with the control group. In addition, overexpression of TBL1XR1 produced a mesenchymal phenotype, while cells with downregulated TBL1XR1 produced an epithelial phenotype. Overexpression of TBL1XR1 significantly increased E-cadherin protein expression whilst snail family transcriptional repressor 1 (SNAI1), zinc finger E-box binding homebox 1 (ZEB1), $\mathrm{p}-\mathrm{Smad} 2 / 3, \operatorname{Smad} 2$ and $\mathrm{Smad} 3$ protein expression was significantly reduced, compared with the control group.
\end{abstract}

Correspondence to: Professor Xiaohua Liang, Department of Oncology, Huashan Hospital, Fudan University, 12 Middle Urumqi Road, Shanghai 200040, P.R. China

E-mail: xhliang66@sina.com

Key words: lung squamous cell carcinomas, transducin $(\beta)$-like 1 $\mathrm{X}$-linked receptor 1, proliferation, invasion, migration, transforming growth factor- $\beta /$ mothers against decapentaplegic homolog signaling pathway, epithelial-mesenchymal transition
Downregulation of TBL1XR1 produced the opposite results. The present study indicated that TBL1XR1 contributed to lung SCC development and progression, and therefore TBL1XR1 may be a potential therapeutic target. TBL1XR1 may induce EMT of lung SCC cells through activation of the TGF- $\beta / S m a d$ signaling pathway.

\section{Introduction}

Lung cancer is the leading cause of cancer mortality and is the most frequently diagnosed cancer in the world (1). Annually, there are an estimated 1.8 million new lung cancer cases, accounting for about $13 \%$ of total cancer diagnoses (2). Historically, lung cancer has been classified as small cell lung cancer and non-small cell lung cancer (NSCLC). NSCLC constitutes $75 \%$ of all lung cancer cases, including adenocarcinoma, squamous cell carcinoma (SCC) and large cell carcinoma. SCC is the largest subset of NSCLC (3). Although surgery and chemoradiotherapy are the primary treatment methods for SCC, the overall 5-year survival rate remains less than $15 \%$ (4). The complex biological characteristics, recurrence, metastasis and low sensitivity to chemotherapeutic drugs remain problematic issues in the treatment of SCC (5-7). Therefore, it is necessary to explore the potential molecular mechanisms underlying the development and progression of SCC, and to identify new therapeutic targets.

Transducin $(\beta)$-like 1 X-linked receptor 1 (TBL1XR1) is an F-box/WD-40-containing protein, a subfamily of F-box proteins that were initially identified as core components of the nuclear receptor corepressor $1 / 2$ complex; this complex serves a role in regulating the activation of corepressors (8-11). Aberrant expression of TBLIXR1 was associated with carcinogenesis and tumor progression by regulating multiple signaling pathways, including the nuclear factor- $\kappa \mathrm{B}$, nuclear receptors, Wnt/ $\beta$-catenin, and Notch pathways (12-15). Studies have previously demonstrated that TBL1XR1 was overexpressed in cervical cancer, nasopharyngeal cancer, breast cancer, hepatocellular carcinoma and esophageal squamous cell cancer, and TBL1XR1 was associated with tumor proliferation, migration, invasion and tumor drug resistance (16-19). Kuang et al (19) found 
that overexpression of TBL1XR1 was associated with the clinicopathological features and prognosis of hepatocellular carcinoma, inducing epithelial-mesenchymal transition (EMT) through the $\mathrm{Wnt} / \beta$-catenin signaling pathway to promote tumor progression.

Although previous studies have demonstrated that TBL1XR1 was highly expressed in human primary lung SCC tissues $(3,20)$, the biological role of TBL1XR1 and its molecular mechanism in lung SCC remain to be established. The present study demonstrated that TBL1XR1 was overexpressed in lung SCC cells. Furthermore, overexpression of TBL1XR1 promoted cell growth, migration, invasion and EMT in lung SCC cells through activation of the TGF- $\beta /$ Smads pathway. These findings suggested that TBL1XR1 serves a role in the progression of lung SCC and may be a potential therapeutic target in lung SCC therapy.

\section{Materials and methods}

Cell lines and cell cultures. The human bronchial epithelial cell line 1 (HBE1) was provided by Xiangya Medical College (Changsha, China) and lung squamous cell carcinoma (SCC) cell lines (SK-MES-1 and H1703) were purchased from Cell Bank of the Chinese Academy of Science (Shanghai, China). Cells were cultured in Dulbecco's modified Eagle medium (DMEM) supplemented with $10 \%$ fetal bovine serum (FBS; both Invitrogen; Thermo Fisher Scientific, Inc., Waltham, MA, USA), $100 \mu \mathrm{g} / \mu \mathrm{l}$ streptomycin and $100 \mu \mathrm{g} / \mu \mathrm{l}$ penicillin, and maintained at $37^{\circ} \mathrm{C}$ in a $5 \% \mathrm{CO}_{2}$-humidified incubator.

Plasmids and small interfering RNAs (siRNAs). The TBL1XR1 plasmid was purchased from Shanghai GeneChem Co., Ltd. (Shanghai, China). The corresponding vector was $\mathrm{pEX}-1$. The TBL1XR1 plasmid and corresponding empty vector were transfected into SK-MES-1 cells using Lipofectamine ${ }^{\circledR} 2000$ reagent (Invitrogen; Thermo Fisher Scientific, Inc.), according to the manufacturer's protocol. Stably transfected cells (SK-MES-1-vector, SK-MES-1-TBL1XR1) were selected by puromycin $(1 \mathrm{ug} / \mathrm{ml}$; InvivoGen, San Diego, CA, USA). TBL1XR1 siRNA sequences and negative control sequences were designed and synthesized by Shanghai GeneChem Co., Ltd. H1703 cells were cultured in six-well plates and transfected with 400 ng TBL1XR1 small interfering (si)RNA (si-TBL1XR1-1, 5'-GCAGCAUAAAGGCCCUAUATT-3'; si-TBL1XR1-2, 5'- GCC UGA UGU A GU ACA A AC AT T-3') using Lipofectamine ${ }^{\circledR} 2000$ reagent (Invitrogen; Thermo Fisher Scientific, Inc.) according to the manufacturer's protocol. Stable cell lines expressing TBL1XR1-siRNA [negative control, (H1703-NC), H1703-siRNA-1, H1703-siRNA-2] were generated with $1 \mathrm{ug} / \mathrm{ml}$ puromycin. Knockdown and overexpression of TBL1XR1 were confirmed by western blot analysis. Western blotting was performed as stated below.

Cell proliferation assay. SK-MES-1-vector, SK-MES-1TBL1XR1, H1703-NC, H1703-siRNA-1 and H1703-siRNA-2 cells were seeded in 96 -well plates at a density of $6 \times 10^{3} /$ well and cultured for 12, 24, 36, 48, 60 and $72 \mathrm{~h}$. Cells were incubated with $100 \mu \mathrm{l}$ of Cell Counting kit-8 (CCK-8) reagent (Dojindo Molecular Technologies, Inc., Kumamoto, Japan) for $2 \mathrm{~h}$ at $37^{\circ} \mathrm{C}$. The absorbance was measured at a wavelength of $450 \mathrm{~nm}$.

Transwell invasion assay. Cell invasion assays were performed using 24-well plates and $8 \mu \mathrm{m}$ Transwell inserts (Corning Life Sciences, Acton, MA, USA). Transwell membrane inserts were precoated with Matrigel ${ }^{\circledR}$ (BD Biosciences, Franklin Lakes, NJ, CA, USA) before adding the cells. A total of $1 \times 10^{5}$ SK-MES-1-vector, SK-MES-1-TBL1XR1, H1703-NC, H1703-siRNA-1, and H1703-siRNA-2 cells in $200 \mu 1$ serum-free DMEM medium were added to the upper chamber. DMEM supplemented with 10\% FBS (500 $\mu \mathrm{l})$ was added to the lower chamber. After incubating the cells at $37^{\circ} \mathrm{C}$ and $5 \% \mathrm{CO}_{2}$ for $48 \mathrm{~h}$ of culture, transfected cells remaining in the upper side of the inserts were removed with cotton swabs. Cells that had migrated to the lower side of the inserts were fixed in methanol and stained with $0.1 \%$ crystal violet for $10 \mathrm{~min}$. Images of migrated cells were captured using an inverted microscope at a magnification, x200. Six visual fields were randomly selected to calculate the number of migrated cells.

Wound healing assay. Transfected cells were cultured in six-well plates until confluent. Straight lines were drawn, in increments of $0.5 \mathrm{~cm}$, on the back of the six-well plates. Cell layers were scratched with a $20 \mu \mathrm{l}$ pipette tip and the medium was replaced with $2 \mathrm{ml}$ of fresh DMEM. Cells were incubated for a further $36 \mathrm{~h}$ at $37^{\circ} \mathrm{C}$. Images were captured at 0 and $36 \mathrm{~h}$ after the scratches were made using an inverted microscope at a magnification, $x 40$. The mean length of the wound was calculated in ImageJ software (version 1.48U; National Institutes of Health, Bethesda, MD, USA) by marking six to eight horizontal lines in the wound.

Western blotting. Total protein was extracted from transfected and non-transfected HBE1, SK-MES-1, H1703 cells using SDS-lysis buffer (Thermo Fisher Scientific, Inc.). Total protein was quantified using the BCA protein assay kit (Thermo Fisher Scientific, Inc.) and protein (20 ug/lane) separated via SDS-PAGE on $10 \%$ gels. The separated proteins were subsequently transferred onto polyvinylidene difluoride membranes, and blocked with 5\% non-fat milk for $1 \mathrm{~h}$ and incubated overnight at $4^{\circ} \mathrm{C}$. Membranes were incubated with the following primary antibodies at 1:1,000 dilution: Anti-TBL1XR1 (cat. no. ab24550; Abcam, Cambridge, UK), anti-GAPDH (cat. no. ab32233; Santa Cruz Biotechnology, Inc., Dallas, TX, USA), anti-snail family transcriptional repressor 1 (SNAI1; cat. no. ab3879), anti-zinc finger E-box binding homebox 1 (ZEB1; cat. no. ab3396), anti-E-calcium-dependent adhesion (E-cadherin; cat. no. ab3195), anti-mothers against decapentaplegic homolog 2 (Smad2; cat. no. ab5339), anti-Smad3 (cat. no. ab9523), anti-phosphorylated (p)-Smad2/3; cat. no. ab8685; all Cell Signaling Technology, Inc., Danvers, MA, USA). Following the primary incubation, membranes were incubated with corresponding horseradish peroxidase-conjugated rabbit anti-mouse and goat anti-rabbit secondary antibodies $(1: 1,000$; cat. no. ab6728 and ab6721, respectively; Abcam) for $1 \mathrm{~h}$ at room temperature. Protein bands were visualized with using the Enhanced 

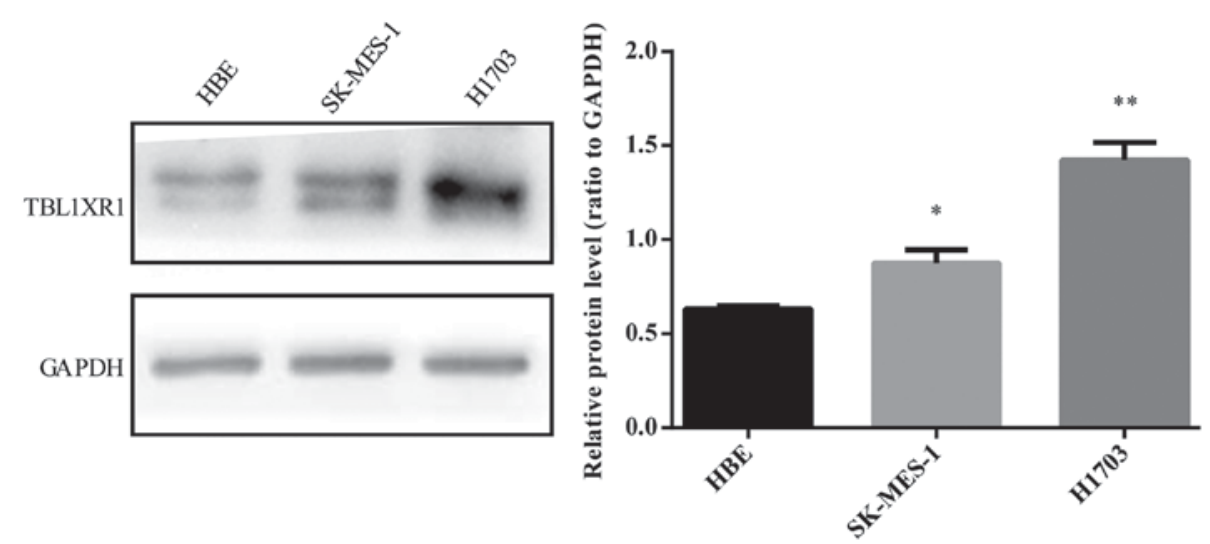

Figure 1. TBL1XR1 protein expression is elevated in lung squamous cell carcinoma cells. The protein expression levels of TBLIXR1 were determined by western blot analysis. The data are presented as the mean \pm standard deviation ${ }^{*} \mathrm{P}<0.05$ and ${ }^{* * *} \mathrm{P}<0.01$ vs. HBE1. TBL1XR1, transducin $(\beta)$-like $1 \mathrm{X}-$ linked receptor 1; HBE1, human bronchial epithelial cell 1 .

Chemiluminescence Western Blotting kit (Thermo Fisher Scientific, Inc.) by exposure to film. Protein expression was quantified using ImageJ software (version 1.48U).

Statistical analysis. All experiments were repeated at least three times and all data expressed as the mean \pm standard error. All statistical analyses were carried out using SPSS software (version 19.0; IBM Corp., Armonk, NY, USA). Differences between two groups were compared using Student's t-tests. For multiple comparisons, one-way analysis of variance was used followed by Turkey's post hoc test. A two-tailed value of $\mathrm{P}<0.05$ was considered to indicate a statistically significant difference.

\section{Results}

TBLIXR1 is highly expressed in human lung SCC cells. To determine the biological role of TBL1XR1 in human lung SCC, TBL1XR1 expression was first examined in lung SCC cell lines (SK-MES-1 and H1703). TBL1XR1 protein expression was examined in SCC cell lines and corresponding control, HBE1 cell line. Protein levels of TBL1XR1 were higher than HBE1 in both SCC cell lines (Fig. 1).

TBL1XR1 promotes lung SCC cell proliferation in vitro. To further confirm the biological functions of TBL1XR1 in lung SCC, TBL1XR1 expression in lung SCC cells was upregulated by transfecting TBL1XR1-expressing vector into SK-MES-1 cells expressing low levels of TBL1XR1 (Fig. 2A). Endogenous TBL1XR1 expression was silenced by transfecting TBL1XR1-siRNA (siRNA-1, siRNA-2) into H1703 cells expressing high levels of TBL1XR1 (Fig. 2B). CCK- 8 assays demonstrated an increase in the number of TBL1XR1-overexpressing cells (SK-MES-1-TBL1XR1) compared with empty vector control cells (SK-MES-1-vector), suggesting that TBL1XR1 overexpression increased the proliferative capacity of lung SCC cells ( $\mathrm{P}<0.05 ; \mathrm{Fig} .2 \mathrm{C})$. Conversely, knockdown of TBL1XR1 (H1703-siRNA-1, H1703-siRNA-2) significantly attenuated lung SCC cell proliferation, leading to a decrease in cell number compared with H1703-NC $(\mathrm{P}<0.05$; Fig. 2D). These results indicate that TBL1XR1 promotes lung SCC cell proliferation in vitro.
TBL1XR1 promotes the migration and invasion of lung SCC cells. The effect of TBL1XR1 on cell migration and invasion ability was analyzed using wound healing and Transwell invasion assays, respectively. The wounds healed better and faster in SK-MES-1-TBL1XR1 cells compared with SK-MES-1-vector cells (Fig. 3A), suggesting that TBL1XR1 enhanced the migration properties of SK-MES-1 cells. Similar results were identified in the Transwell invasion assays where overexpression of TBL1XR1 significantly increased the invasion ability of SK-MES-1 cells (Fig. 3B). The wound healing assays and Transwell invasion assays demonstrated that TBL1XR1 knockdown significantly decreased the migration and invasion ability of H1703 cells (Fig. 3C and D). These results suggest that TBL1XR1 may promote lung SCC cell migration and invasion in vitro.

TBLIXR1 promotes EMT in lung SCC cells. To investigate the molecular mechanism of TBL1XR1 in the regulation of lung SCC cellular migration and invasion, cell morphology was analyzed and the levels of EMT markers were tested. The morphology of TBL1XR1-transfected lung SCC cells appeared fusiform with the formation of protuberances associated with a mesenchymal phenotype (21), compared with vector control cells (Fig. 4A). TBL1XR1 knockdown reduced the number of irregular branched structures in both H1703-siRNA cells. The cell morphology appeared to be short shuttle-like, with some round-shaped cells associated with an epithelial phenotype, compared with vector control cells (Fig. 4B). Western blotting revealed that overexpression of TBL1XR1 significantly decreased the expression level of the epithelial cell marker E-cadherin and increased the expression levels of ZEB1 transcription factor (Fig. 5). SNAI1, a stimulator of EMT in tumors (22), was elevated in SK-MES-1-TBL1XR1 cells (Fig. 5A-a, b, c, g). By contrast, downregulation of TBL1XR1 significantly increased the expression level of E-cadherin and decreased expression levels of both ZEB1 and SNAI1 (Fig. 5B-a, b, c, g). Taken together, these results suggest that TBL1XR1 may induce EMT in lung SCC cells in vitro.

TBL1XR1 induces EMT the through TGF- $\beta /$ Smad signaling pathway. Previous studies revealed that the TGF- $\beta /$ Smad 

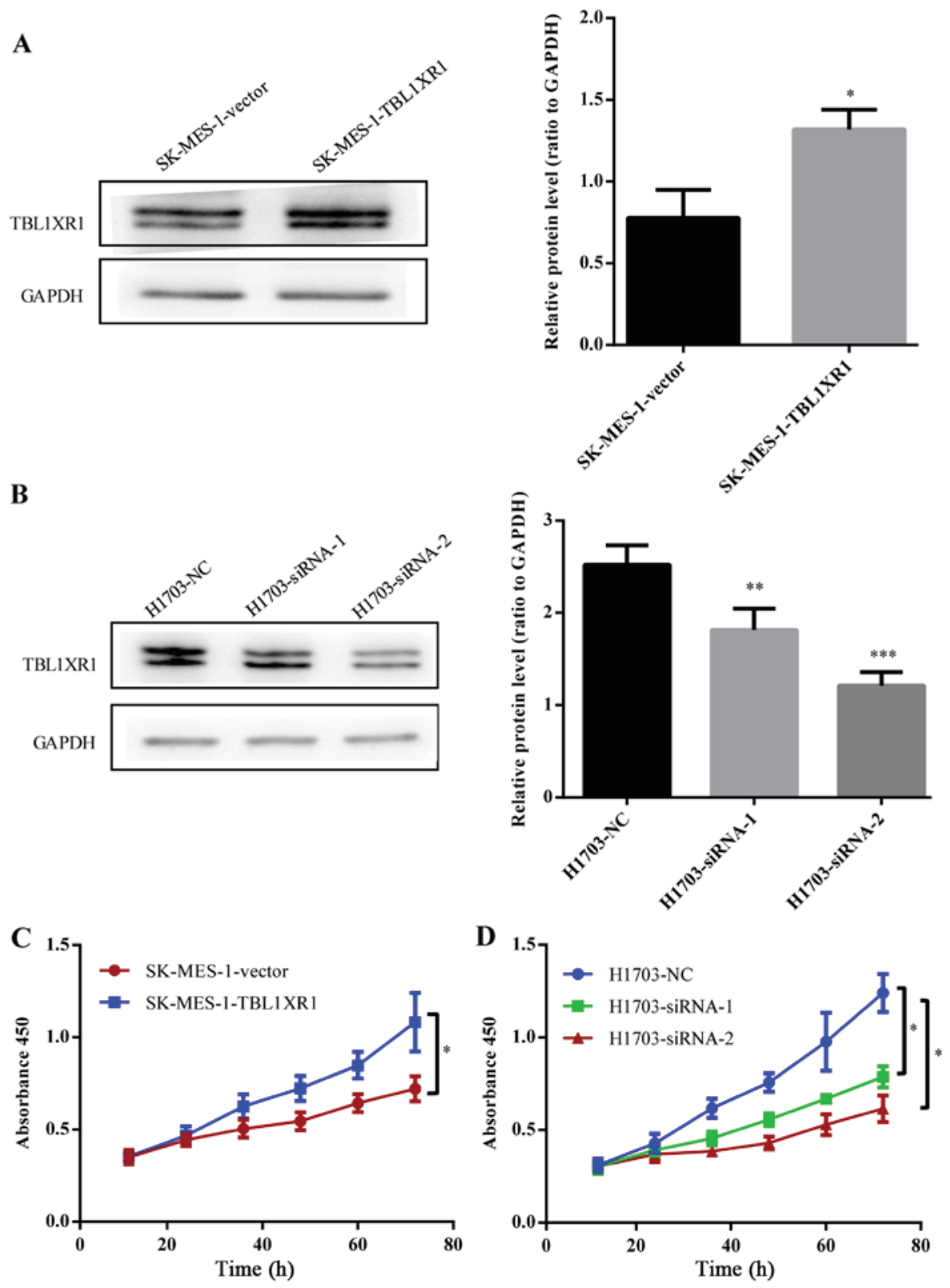

Figure 2. TBL1XR1 promotes cell proliferation of lung SCC in vitro. (A) TBL1XR1 protein expression was determined by western blot analysis in SK-MES-1 cells treated with TBL1XR1-expressing vector. ${ }^{\text {P }}<0.05$ vs. SK-MES-1-vector. (B) TBLIXR1 protein expression was determined by western blot analysis in H1703 cells treated with TBL1XR1-siRNA (siRNA-1, siRNA-2) ${ }^{* *} \mathrm{P}<0.01$ and ${ }^{* * *} \mathrm{P}<0.001$ vs. H1703-NC. (C) The effect of TBL1XR1 overexpression on SK-MES-1 cell growth was analyzed by CCK- 8 assay. "P<0.05 as indicated. (D) The effect of TBL1XR1 knockdown on H1703 cell growth was analyzed by CCK- 8 assay. ${ }^{*} \mathrm{P}<0.05$ as indicated. The data are presented as the mean \pm standard deviation three independent experiments. CCK- 8 , cell counting kit- 8 ; TBL1XR1, transducin ( $\beta$ )-like 1 X-linked receptor 1; siRNA, small interfering RNA; H1703-NC and SK-MES-1-vector, negative transfection controls; SK-MES-1-TBL1XR1, TBL1XR1-overexpressing SK-MES-1 cells; H1703-siRNA-1 and H1703-siRNA-2, TBL1XR1 knockdown in H1703 cells.

signaling pathway served a key role in the process of tumor cell migration and invasion via the induction of EMT (23-25). Another study demonstrated that TGF- $\beta$-induced EMT in non-small cell lung cancer (26). The present study evaluated whether activation of the TGF- $\beta /$ Smad pathway was involved in TBL1XR1-induced EMT in lung SCC. Western blot analysis revealed that expression levels of $\mathrm{p}-\mathrm{Smad} 2 / 3, \operatorname{Smad} 2$ and Smad3 were significantly increased in SK-MES-1-TBL1XR1 cells whilst these expression levels significantly decreased following the knockdown of TBL1XR1 in lung SCC cells (H1703-siRNA-1, H1703-siRNA-2), compared with the H1702-NC group respectively (Fig. 5A-d, e,f, g and B-d, e, f, g). Therefore, TBL1XR1 appears to regulate EMT to induce lung
SCC cell proliferation and invasion via the TGF- $\beta / \mathrm{Smad}$ signaling pathway.

\section{Discussion}

TBL1XR1 is a transcriptional cofactor involved in controlling the switch between gene activation and repression in transcriptional regulation (12). Abnormal TBLIXR1 expression is associated with the occurrence and development of malignant tumors $(27,28)$. TBLIXR1 inhibited the growth of prostate cancer by selectively activating androgen receptor target genes (27). Furthermore, one study indicated that TBLIXR1 promoted proliferation and tumorigenicity in breast cancer 
A

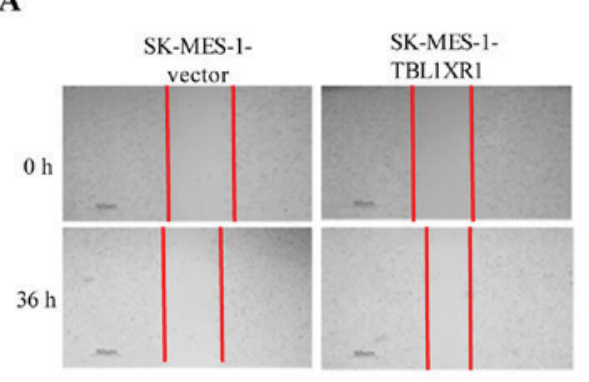

B

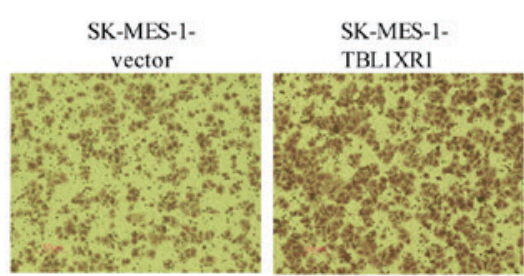

C

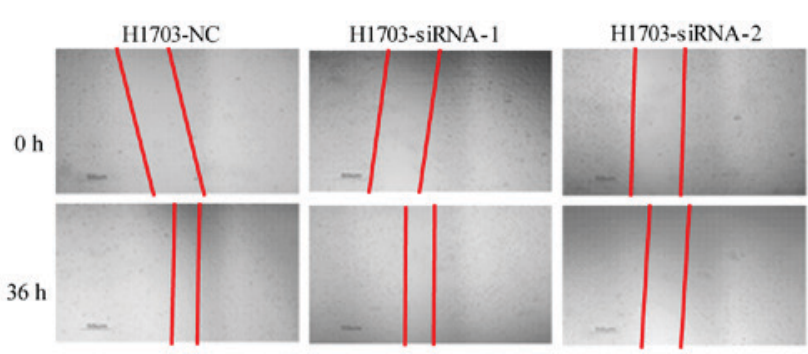

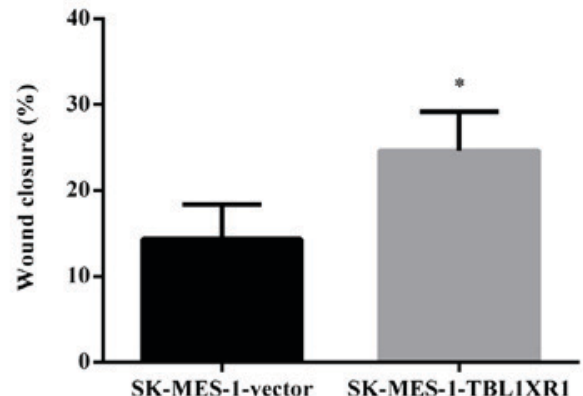

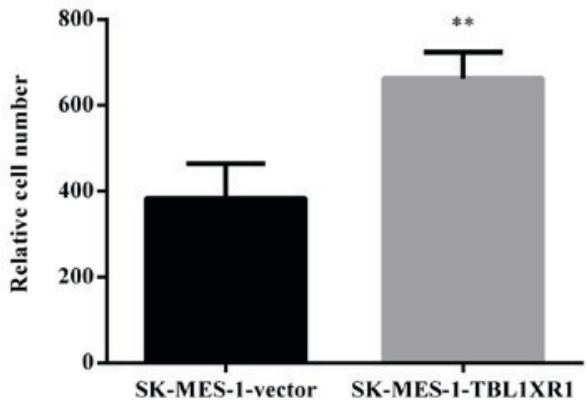

SK-MES-1-vector SK-MES-1-TBL1XR1

D
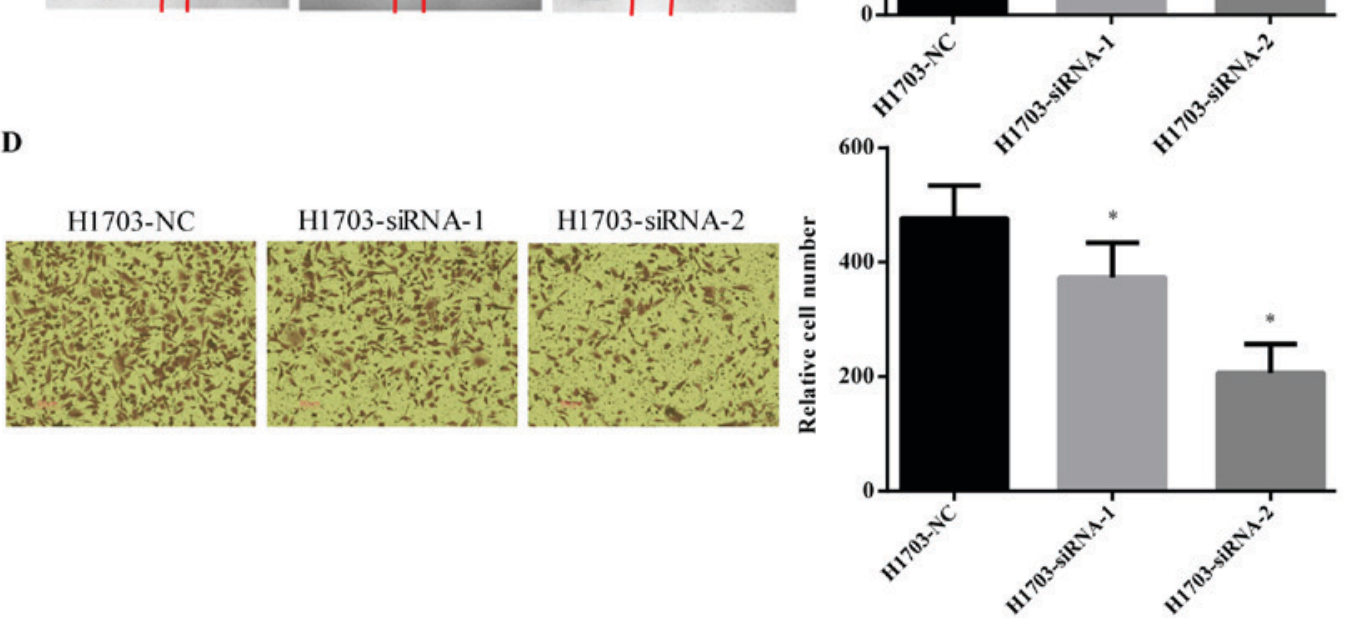

Figure 3. TBL1XR1 promotes the migratory and invasive ability of lung SCC cells. (A) Effects of TBL1XR1 overexpression on SK-MES-1 cell migration rates were determined using wound healing assays in vitro. Observations were recorded at 0 and $36 \mathrm{~h}$ after scratching a confluent layer of cells (magnification, $\mathrm{x} 40$ ). ${ }^{*} \mathrm{P}<0.05$ vs. SK-MES-1-vector. (B) Effects of TBL1XR1 overexpression on SK-MES-1 cell invasion was determined using the Transwell invasion assay with chambers precoated with Matrigel ${ }^{\circledR}$ (magnification, $\mathrm{x} 200$ ). ${ }^{* *} \mathrm{P}<0.01$ vs. SK-MES-1-vector. (C) Effects of TBL1XR1 knockdown on H1703 cell migration rates were determined using wound healing assays in vitro. Observations were recorded at 0 and $36 \mathrm{~h}$ after scratching a confluent layer of cells (magnification, $\mathrm{x} 40$ ). "P<0.05 vs. H1703-NC. (D) Effects of TBL1XR1 overexpression on SK-MES-1 cell invasion was determined using the Transwell invasion assay with chambers precoated with Matrigel ${ }^{\circledast}$ (magnification, $\mathrm{x} 200$ ). ${ }^{*} \mathrm{P}<0.05$ vs. H1703-NC. The data are presented as the mean \pm standard deviation three independent experiments. TBL1XR1, transducin ( $\beta$ )-like 1 X-linked receptor 1; siRNA, small interfering RNA; H1703-NC and SK-MES-1-vector, negative transfection controls; SK-MES-1-TBL1XR1, TBL1XR1-overexpressing SK-MES-1 cells; H1703-siRNA-1 and H1703-siRNA-2, TBL1XR1 knockdown in H1703 cells.

by activating genes downstream of $\beta$-catenin (17). Previous studies demonstrated that TBL1XR1 was overexpressed in human primary lung SCC cells (20). However, the exact role of TBL1XR1 in lung SCC remained unexplored. Consistent with previous studies, the data presented in the current study demonstrated that TBL1XR1 protein expression was higher in lung SCC cells compared with human bronchial epithelial cell lines. Furthermore, overexpression of TBL1XR1 promoted the proliferation, invasion and migration of lung SCC cells in vitro, while knockdown of TBL1XR1 significantly inhibited tumorigenicity, by promoting invasion and migration, and proliferation in lung SCC cells. Taken together, the data presented in this study suggested an oncogenic function of TBL1XR1 in the development and progression of lung SCC. 

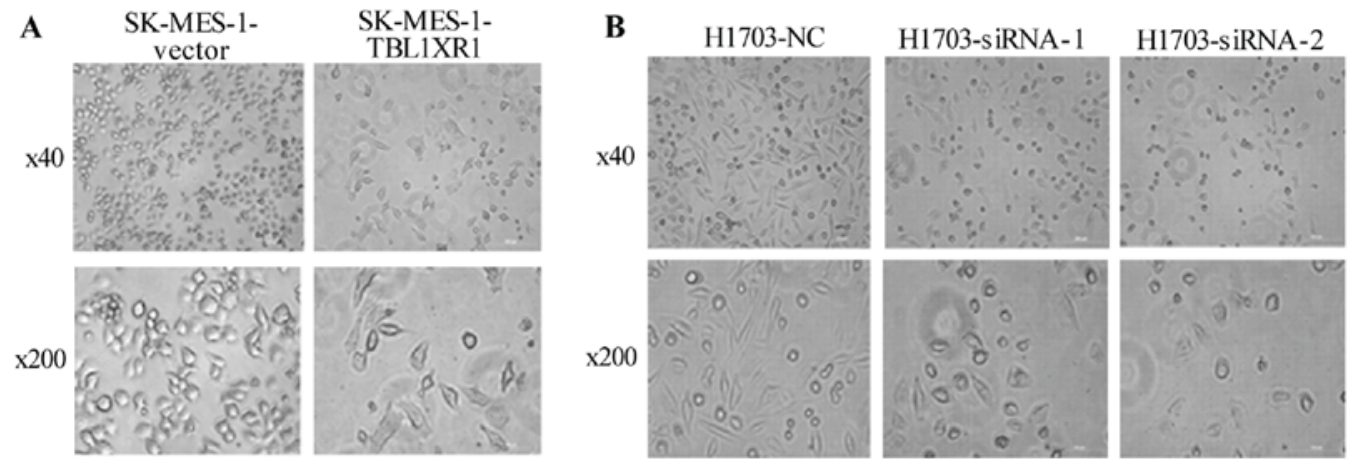

Figure 4. TBL1XR1 promotes an EMT phenotype in lung SCC cells. The effects of TBL1XR1 (A) overexpression and (B) knockdown on EMT were examined by observing morphological changes in in lung SCC cells (magnification, $\mathrm{x} 40$ and x200). EMT, epithelial-mesenchymal transition; TBL1XR1, transducin ( $\beta$ )-like 1 X-linked receptor 1; siRNA, small interfering RNA; H1703-NC and SK-MES-1-vector, negative transfection controls; SK-MES-1-TBL1XR1, TBL1XR1-overexpressing SK-MES-1 cells; H1703-siRNA-1 and H1703-siRNA-2, TBL1XR1 knockdown in H1703 cells; SCC, squamous cell carcinoma.

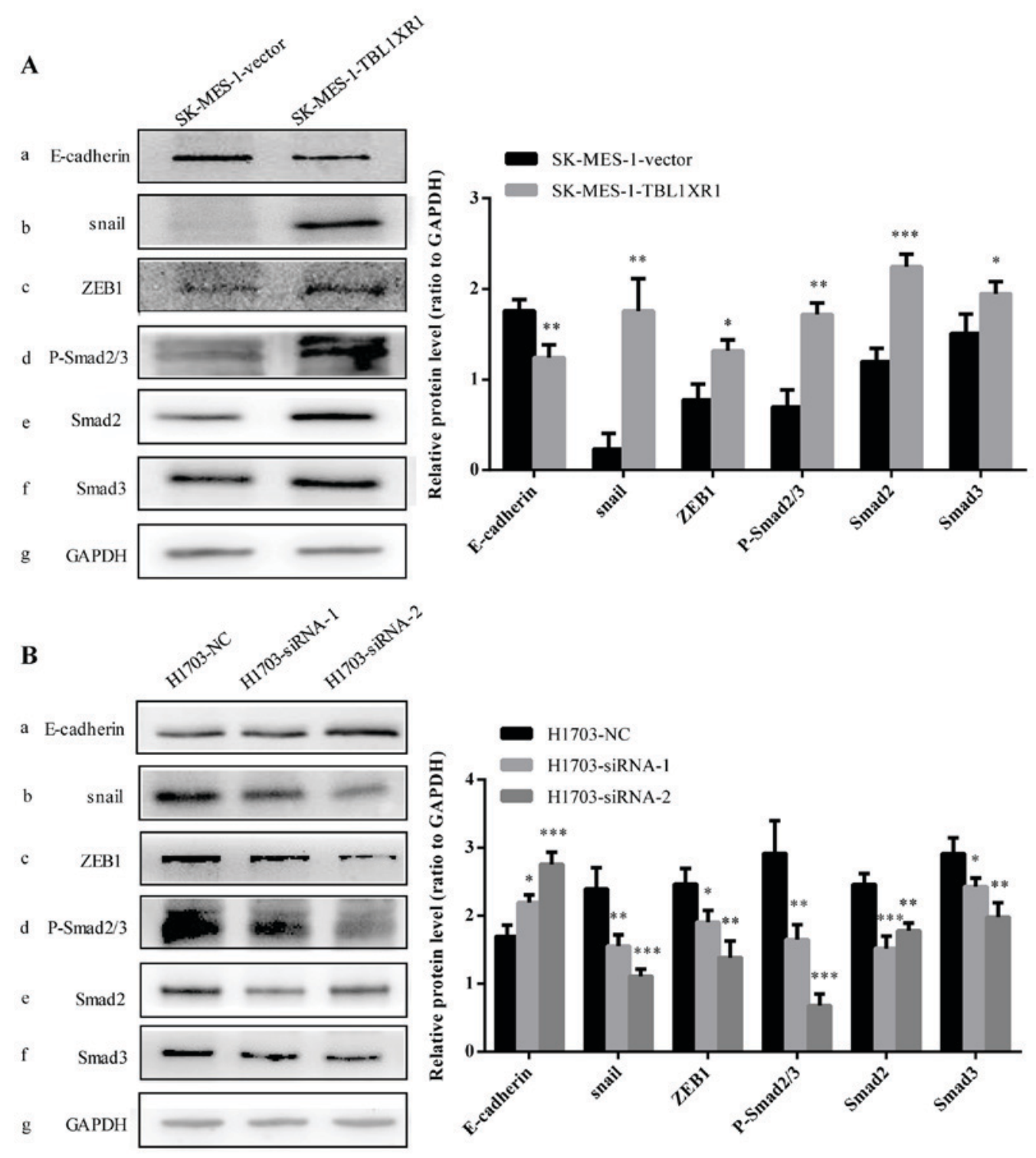

Figure 5. TBL1XR1-induced EMT is mediated via the TGF- $\beta /$ Smad signaling pathway. The protein expression levels of (A-a) E-cadherin, (A-b) SNAI1, (A-c) ZEB1, (A-d) p-Smad2/3, (A-e) Smad2 and (A-f) Smad3 were determined using western blot analysis in SK-MES-1 cells following overexpression of TBL1XR1. GAPDH was used as the loading control. ${ }^{*} \mathrm{P}<0.05,{ }^{* * *} \mathrm{P}<0.01$ and ${ }^{* * * *} \mathrm{P}<0.001$ vs. SK-MES-1-vector. The protein expression levels of (B-a) E-cadherin, (B-b) SNAI1, (B-c) ZEB1, (B-d) p-Smad2/3, (B-e) Smad2, and (B-f) Smad3 in H1703 cells following TBLIXR1 knockdown. GAPDH was used as the loading control. ${ }^{*} \mathrm{P}<0.05,{ }^{* *} \mathrm{P}<0.01$ and ${ }^{* * * *} \mathrm{P}<0.001$ vs. H1703-NC. EMT, epithelial-mesenchymal transition; TBL1XR1, transducin ( $\beta$ )-like $1 \mathrm{X}$-linked receptor 1; TGF- $\beta /$ Smad, transforming growth factor $\beta /$ mothers against decapentaplegic homolog; p-Smad2/3, phosphorylated Smad2 and Smad3; siRNA, small interfering RNA; ZEB1, zinc finger E-box binding homeobox 1; H1703-NC and SK-MES-1-vector, negative transfection controls; SK-MES-1-TBL1XR1, TBL1XR1-overexpressing SK-MES-1 cells; H1703-siRNA-1 and H1703-siRNA-2, TBL1XR1 knockdown in H1703 cells. 
EMT, a process in which epithelial cells differentiate into mesenchymal cells in response to a number of physiological and pathological conditions, serves an important role in tumor invasion and metastasis (29). In the EMT process, migratory and invasive capabilities are acquired through the loss of polarity and cell-cell adhesion of the epithelial cells, and as a result, cell morphology is transformed from the low-invasive epithelial phenotype to the high-invasive mesenchymal phenotype (30). Cell surface markers change correspondingly, with the downregulation of the epithelial marker E-cadherin and the upregulation of the mesenchymal marker N-cadherin (31). Various transcription factors, including SNAI1, twist-related protein 1 and ZEB1 are also upregulated (30,31-33). The present study revealed that TBL1XR1-induced lung SCC cells undergo morphological alterations caused by decreased expression of E-cadherin and increased expression of SNAI1 and ZEB1. These findings suggested that TBL1XR1 may promote lung SCC aggressiveness by inducing EMT, and, therefore, TBL1XR1 may be a potential therapeutic target in lung SCC therapy.

It has been reported that EMT occurs by activation of several signaling pathways, including phosphoinositide 3-kinase/protein kinase B (Akt), TGF//Smad, integrin-linked protein kinase/Akt, and Wnt- $\beta$-catenin, which activate E-cadherin repressors of the Snail family (34). TGF $\beta$-Smad is one of the most important signaling pathways contributing to EMT, which involves the binding of TGF- $\beta$ to its receptor (TGF- $\beta$ RI/TGF- $\beta$ RII) and the subsequent phosphorylation, leading to Smad2/3 activation $(35,36)$. Activated Smad2/3 forms complexes with Smad4 in the cytoplasm, moving into the nucleus where transcription factors regulate the transcription of target genes (37). SNAI1 is a TGF- $\beta /$ Smad signaling pathway-mediated gene that promotes EMT by repressing E-cadherin expression and increasing invasion and metastasis of tumor cells (25). The current study demonstrated that overexpression of TBL1XR1 increased the expression of SNAI1 and ZEB1 transcription factors, and p-Smad2/3, Smad2 and Smad3 proteins. Downregulation of TBL1XR1 gave the opposite results. The present study indicated that TBL1XR1 may induce EMT of lung SCC cells through activation of the TGF- $\beta / \mathrm{Smad}$ signaling pathway to promote the development of lung SCC.

Although the present study provided some understanding of TBL1XR1, certain limitations should be noted. TBL1XR1 was detected at a cellular level, and, therefore, an additional histological study would be required to validate the results. Furthermore, the current study indicated that TBL1XR1 may induce EMT of lung SCC cells by activating the TGF- $\beta / \mathrm{Smad}$ signaling pathway. Due to a lack of TGF- $\beta$ inhibitor treatment group, definite conclusions cannot be drawn and further investigation is needed to verify this effect.

In conclusion, this preliminary study identified a biological role for TBL1XR1 and possible molecular mechanisms in lung SCC. First, TBL1XR1 expression was elevated in lung SCC cells. Second, TBL1XR1 promoted proliferation, invasion and migration of lung SCC cells in vitro. Finally, TBL1XR1 may induce EMT of lung SCC cells through activation of the TGF- $\beta /$ Smad signaling pathway.

\section{Acknowledgements}

Not applicable.

\section{Funding}

No funding was received.

\section{Availability of data and materials}

All data generated or analyzed during this study are included in the published article.

\section{Authors' contributions}

YZ, HL, and MG performed experimental work. YZ and JJ analyzed the data. YZ wrote the manuscript. XL designed the study and revised the manuscript.

\section{Ethics approval and consent to participate}

The present study was approved by the Ethics Committee of the Huashan Hospital of Fudan University (Shanghai, China) and informed consent was taken from all patients.

\section{Patient consent for publication}

Not applicable.

\section{Competing interests}

The authors declare that they have no competing interests.

\section{References}

1. Tanoue LT, Tanner NT, Gould MK and Silvestri GA: Lung cancer screening. Am J Respir Crit Care Med 191: 19-33, 2015.

2. Torre LA, Bray F, Siegel RL, Ferlay J, Lortet-Tieulent J and Jemal A: Global cancer statistics, 2012. CA Cancer J Clin 65: 87-108, 2015

3. An Q, Pacyna-Gengelbach M, Schlüns K, Deutschmann N, Guo S, Gao Y, Zhang J, Cheng S and Petersen I: Identification of differentially expressed genes in immortalized human bronchial epithelial cell line as a model for in vitro study of lung carcinogenesis. Int J Cancer 103: 194-204, 2003.

4. Cheng TD, Cramb SM, Baade PD, Youlden DR, Nwogu C and Reid ME: The international epidemiology of lung cancer: Latest trends, disparities, and tumor characteristics. J Thorac Oncol 11: 1653-1671,2016.

5. Liao BC, Shao YY, Chen HM, Shau WY, Lin ZZ, Kuo RN, Lai CL, Chen KH, Cheng AL, Yang JC and Lai MS: Comparative effectiveness of first-line platinum-based chemotherapy regimens for advanced lung squamous cell carcinoma. Clin Lung Cancer 16: 137-143, 2015.

6. Travis WD: Pathology of lung cancer. Clin Chest Med 32: 669-692, 2011

7. Mizoguchi K, Nakamura Y, Sano K, Sato S, Ikegami Y, Motoshima K, Takemoto S, Ogawara D, Senju H, Sugasaki N, et al: Pharmacokinetic parameters of gefitinib predict efficacy and toxicity in patients with advanced non-small cell lung cancer harboring EGFR mutations. Cancer Chemother Pharmacol 78: 377-382, 2016.

8. Zhang X, Dormady SP and Basch RS: Identification of four human cDNAs that are differentially expressed by early hematopoietic progenitors. Exp Hematol 28: 1286-1296, 2000.

9. Andersson S, Wallin KL, Hellström AC, Morrison LE, Hjerpe A, Auer G, Ried T, Larsson C and Heselmeyer-Haddad K: Frequent gain of the human telomerase gene TERC at $3 q 26$ in cervical adenocarcinomas. Br J Cancer 95: 331-338, 2006.

10. Yang YC, Shyong WY, Chang MS, Chen YJ, Lin CH, Huang ZD, Wang, Hsu MT and Chen ML: Frequent gain of copy number on the long arm of chromosome 3 in human cervical adenocarcinoma. Cancer Genet Cytogenet 131: 48-53, 2001. 
11. Zhang J, Kalkum M, Chait BT and Roeder RG: The N-CoR-HDAC3 nuclear receptor corepressor complex inhibits the JNK pathway through the integral subunit GPS2. Mol Cell 9: 611-623, 2002

12. Perissi V, Aggarwal A, Glass CK, Rose DW and Rosenfeld MG: A corepressor/coactivator exchange complex required for transcriptional activation by nuclear receptors and other regulated transcription factors. Cell 116: 511-526, 2004.

13. Choi HK, Choi KC, Yoo JY, Song M, Ko SJ, Kim CH, Ahn JH, Chun KH, Yook JI and Yoon HG: Reversible SUMOylation of TBL1-TBLR1 regulates $\beta$-catenin-mediated Wnt signaling. Mol Cell 43: 203-216, 2011.

14. Hoberg JE, Yeung F and Mayo MW: SMRT derepression by the IkappaB kinase alpha: A prerequisite to NF-kappaB transcription and survival. Mol Cell 16: 245-255, 2004.

15. Li J and Wang CY: TBL1-TBLR1 and beta-catenin recruit each other to Wnt target-gene promoter for transcription activation and oncogenesis. Nat Cell Biol 10: 160-169, 2008.

16. Wang J, Ou J, Guo Y, Dai T, Li X, Liu J, Xia M, Liu L and He M: TBLR1 is a novel prognostic marker and promotes epithelial-mesenchymal transition in cervical cancer. Br J Cancer 111: $112-124,2014$.

17. Li X,Liang W, Liu J,Lin C, Wu S, Song L and Yuan Z: Transducin $(\beta)$-like $1 \mathrm{X}$-linked receptor 1 promotes proliferation and tumorigenicity in human breast cancer via activation of beta-catenin signaling. Breast Cancer Res 16: 465, 2014.

18. Chen SP, Yang Q, Wang CJ, Zhang LJ, Fang Y, Lei FY, Wu S, Song LB, Guo X and Guo L: Transducin $\beta$-like $1 \mathrm{X}$-linked receptor 1 suppresses cisplatin sensitivity in nasopharyngeal

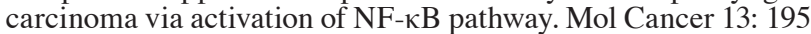
2014.

19. Kuang X, Zhu J, Peng Z, Wang J and Chen Z: Transducin (Beta)-like $1 \mathrm{X}$-linked receptor 1 correlates with clinical prognosis and epithelial-mesenchymal transition in hepatocellular carcinoma. Dig Dis Sci 61: 489-500, 2016.

20. Liu Y, Sun W, Zhang K, Zheng H, Ma Y, Lin D, Zhang X, Feng L, Lei $\mathrm{W}$, Zhang $\mathrm{Z}$, et al: Identification of genes differentially expressed in human primary lung squamous cell carcinoma. Lung Cancer 56: 307-317, 2007.

21. Yan J, Gumireddy K, Li A and Huang Q: Regulation of mesenchymal phenotype by MicroRNAs in cancer. Curr Cancer Drug Targets 13: 930-934, 2013.

22. Giannelli G, Bergamini C, Fransvea E, Sgarra C and Antonaci S Laminin-5 with transforming growth factor-beta1 induces epithelial to mesenchymal transition in hepatocellular carcinoma. Gastroenterology 129: 1375-1383, 2005.

23. Li N, Xu H, Fan K, Liu X, Qi J, Zhao C, Yin P, Wang L, Li Z and Zha X: Altered beta1,6-GlcNAc branched N-glycans impair TGF- $\beta$-mediated epithelial-to-mesenchymal transition through Smad signalling pathway in human lung cancer. J Cell Mol Med 18: 1975-1991, 2014.

24. Yang G, Liang Y, Zheng T, Song R, Wang J, Shi H, Sun B, Xie C, Li Y, Han J, et al: FCN2 inhibits epithelial-mesenchymal transition-induced metastasis of hepatocellular carcinoma via TGF- $\beta /$ Smad signaling. Cancer Lett 378: 80-86, 2016.
25. Ji Q, Liu X, Han Z, Zhou L, Sui H, Yan L, Jiang H, Ren J, Cai J and Li Q: Resveratrol suppresses epithelial-to-mesenchymal transition in colorectal cancer through TGF- $\beta 1 /$ Smads signaling pathway mediated Snail/E-cadherin expression. BMC Cancer 15: 97, 2015.

26. Li C, Wan L, Liu Z, Xu G, Wang S, Su Z, Zhang Y, Zhang C, Liu X, Lei Z and Zhang HT: Long non-coding RNA XIST promotes TGF- $\beta$-induced epithelial-mesenchymal transition by regulating miR-367/141-ZEB2 axis in non-small-cell lung cancer. Cancer Lett 418: 185-195, 2018.

27. Daniels G, Li Y, Gellert LL, Zhou A, Melamed J, Wu X, Zhang X, Zhang D, Meruelo D, Logan SK, et al: TBLR1 as an androgen receptor (AR) coactivator selectively activates AR target genes to inhibit prostate cancer growth. Endocr Relat Cancer 21: 127-142, 2013.

28. Liu F, He Y, Cao Q, Liu N and Zhang W: TBL1XR1 is highly expressed in gastric cancer and predicts poor prognosis. Dis Markers 2016: 2436518, 2016.

29. Thiery JP, Acloque H, Huang RY and Nieto MA Epithelial-mesenchymal transitions in development and disease. Cell 139: 871-890, 2009.

30. Lamouille S, Xu J and Derynck R: Molecular mechanisms of epithelial-mesenchymal transition. Nat Rev Mol Cell Bio 15: 178-196, 2014.

31. Zhai B, Yan HX, Liu SQ, Chen L, Wu MC and Wang HY: Reduced expression of E-cadherin/catenin complex in hepatocellular carcinomas. World J Gastroentero 14: 5665-5673, 2008.

32. Zhai X, Zhu H, Wang W, Zhang S, Zhang Y and Mao G: Abnormal expression of EMT-related proteins, S100A4, vimentin and E-cadherin, is correlated with clinicopathological features and prognosis in HCC. Med Oncol 31: 970, 2014

33. Lee TK, Poon RT, Yuen AP, Ling MT, Kwok WK, Wang XH, Wong YC, Guan XY, Man K, Chau KL and Fan ST: Twist overexpression correlates with hepatocellular carcinoma metastasis through induction of epithelial-mesenchymal transition. Clin Cancer Res 12: 5369-5376, 2006.

34. Guarino M: Epithelial-mesenchymal transition and tumour invasion. Int J Biochem Cell Biol 39: 2153-2160, 2007.

35. Hu H, Wang M, Wang H, Liu Z, Guan X, Yang R, Huang R, Tang $\mathrm{Q}$, Zou $\mathrm{C}$, Wang $\mathrm{G}$, et al: MEGF6 promotes the epithelial-to-mesenchymal transition via the TGF/SMAD signaling pathway in colorectal cancer metastasis. Cell Physiol Biochem 46: 1895-1906, 2018.

36. Jung B, Staudacher JJ and Beauchamp D: Transforming growth factor $\beta$ superfamily signaling in development of colorectal cancer. Gastroenterology 152: 36-52, 2017.

37. Tsang KJ, Tsang D, Brown TN and Crowe DL: A novel dominant negative Smad 2 mutation in a TGFbeta resistant human carcinoma cell line. Anticancer Res 22: 13-19, 2002.

(i)(9) This work is licensed under a Creative Commons Attribution-NonCommercial-NoDerivatives 4.0 International (CC BY-NC-ND 4.0) License. 